\title{
Gastric Carcinoma: An Unexpected Complication of Loop Gastro-Jejunostomy Done in Childhood
}

Sri Hari Priya Vemulakonda ${ }^{1}$, Rehena Sulthana ${ }^{1}$, Ankit Jain ${ }^{1}$, Abhinaya Reddy ${ }^{1}$, Vishnu Prasad Nelamangala Ramakrishnaiah ${ }^{1}$

1. Surgery, Jawaharlal Institute of Postgraduate Medical Education and Research, Puducherry, IND

Corresponding author: Vishnu Prasad Nelamangala Ramakrishnaiah, vprasad285@gmail.com

\begin{abstract}
Gastric adenocarcinoma is the fifth most common cancer worldwide and the third leading cause of death. The major risk factors include Helicobacter pylori infection, genetic factors, environmental factors, and atrophic gastritis. Gastric remnant cancer is gastric carcinoma that develops in the remnant stomach more than five years after distal gastrectomy for benign disease, incidence ranging from $1 \%$ to $8 \%$. However, gastric carcinoma after loop gastro-jejunostomy without gastric resection for benign etiology is rare. We report a case of a 45 -year-old lady with gastro-jejunostomy without gastric resection done in childhood, presenting with adenocarcinoma at the anastomotic site after 35 years.
\end{abstract}

Categories: Gastroenterology, General Surgery, Oncology

Keywords: gastric carcinoma, gastro-jejunostomy, biliary reflux, adenocarcinoma, gastrectomy

\section{Introduction}

Gastric cancer is the fifth most common cancer worldwide and the third leading cause of death [1]. Adenocarcinoma of the stomach is the most common type. Helicobacter pylori and Epstein Barr virus infection, dietary factors like smoked and salted food consumption, environmental factors like poor socioeconomic status, atrophic gastritis, and adenomatous polyps are some of the risk factors for developing gastric carcinoma. However, gastric carcinoma after Billroth-II type loop gastro-jejunostomy (GJ) for benign etiology is rare [2]. Here, we report a case of a 45 -year-old lady who presented with gastric adenocarcinoma following GJ done in childhood.

Review began 06/03/2021 Review ended 06/09/2021 Published 06/24/2021

\section{Copyright 2021}

Vemulakonda et al. This is an open access article distributed under the terms of the Creative Commons Attribution License CC-BY 4.0., which permits unrestricted use, distribution, and reproduction in any medium, provided the original author and source are credited.

\section{Case Presentation}

A 45-year-old lady presented to the emergency with multiple episodes of non-bilious vomiting, containing undigested old food particles, for the past two months. The vomitus was associated with epigastric pain, on and off post-prandial abdominal distension, early satiety, decreased appetite, and significant weight loss over the past two months. However, there was no history of hematemesis, melena, or jaundice. The patient gave a history of an upper abdominal surgery in her childhood (around 35 years ago), details of which were not known, and no records were available. At presentation, she had tachycardia $(110 / \mathrm{min})$ and was dehydrated. The abdomen was soft with distension of the upper abdomen, and the succussion splash was positive. There was an upper midline vertical scar present, healed with primary intention. Blood investigations showed hemoglobin of $7.7 \mathrm{~g} / \mathrm{dL}$, urea of $12 \mathrm{mg} / \mathrm{dL}$, creatinine of $0.28 \mathrm{mg} / \mathrm{dL}$, sodium of 129 $\mathrm{mEq} / \mathrm{L}$, and potassium of $4.2 \mathrm{mEq} / \mathrm{L}$.

Upper gastrointestinal endoscopy (UGIE) revealed an ulcero-proliferative circumferential growth involving the lesser curvature, body, and the antro-pyloric region of the stomach. Multiple biopsies taken from the growth were suggestive of gastric adenocarcinoma. Contrast-enhanced computed tomography (CECT) revealed a circumferential growth involving the body and antro-pyloric region of the stomach. The growth was adherent to bowel loops with loss of fat planes with liver and pancreas. She was taken up for staging laparoscopy. Due to the absence of liver or peritoneal metastasis, and no infiltration of the growth into the liver or pancreas, we proceeded with total gastrectomy. However, growth was found adherent to the jejunal loops traversing through the transverse mesocolon. On further dissection, the adherent jejunal loop was found to be retro-colic posterior loop GJ (Figures 1A, 1B). 


\section{Cureus}

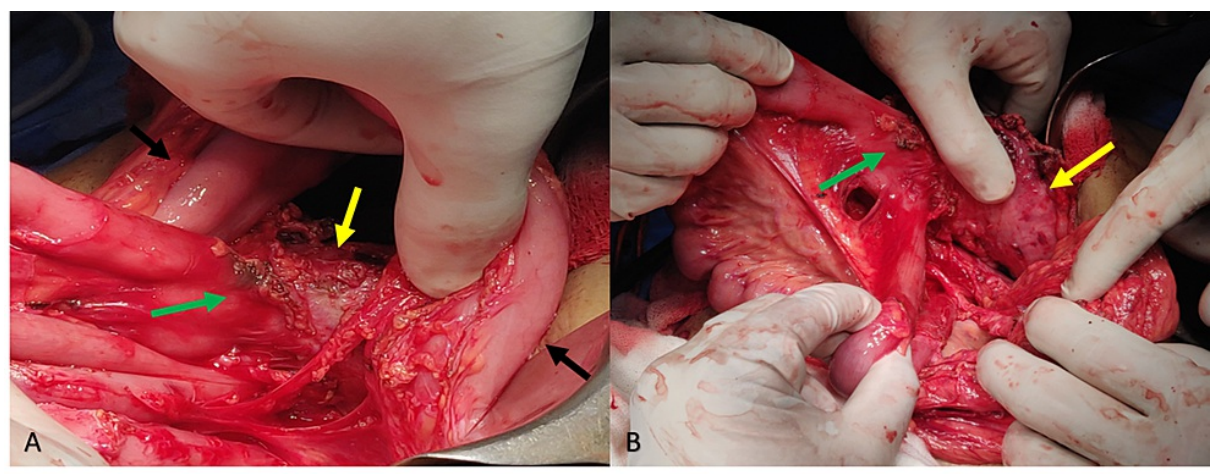

FIGURE 1: (A, B) Intraoperative images showing the retro-colic gastrojejunostomy to the posterior wall of the stomach. (Black arrow: Transverse colon. Yellow arrow: Stomach. Green arrow: Gastrojejunostomy site.)

Total gastrectomy with excision of previous GJ loops for a length of $10 \mathrm{~cm}$ along the afferent and efferent jejunal loop was done (Figure 2).

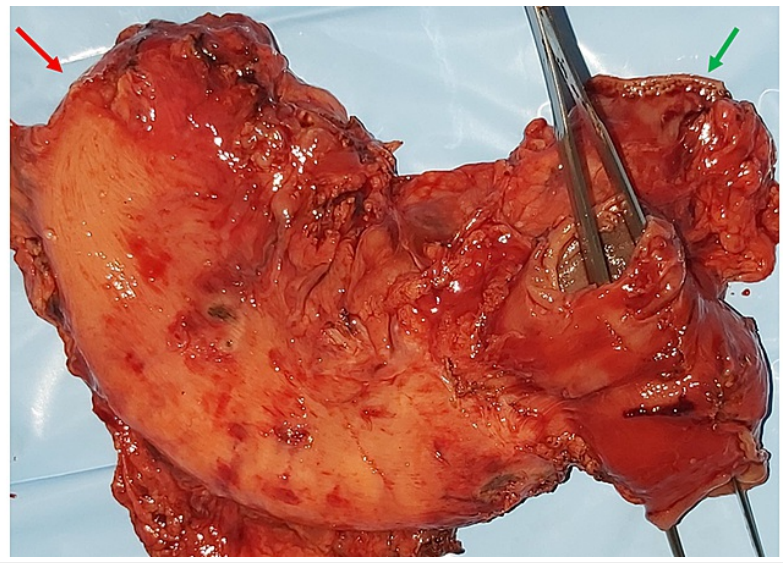

FIGURE 2: Resected specimen showing the posterior surface of the stomach with forceps passed through the jejunal loop of gastrojejunostomy. (Red arrow- Stapled esophageal end of the stomach. Green arrow- Stapled duodenal end of the stomach.)

Intestinal continuity was restored by Roux-en-Y esophago-jejunostomy and jejuno-jejunostomy. The patient had an uneventful postoperative recovery and was started on capecitabine-based adjuvant chemotherapy.

\section{Discussion}

Except for malignant conditions, loop GJ is commonly done for benign gastric outlet obstruction (GOO) due to peptic stricture, caustic ingestion, inflammatory diseases such as Crohn's disease or tuberculosis or as part of surgical procedures such as mini gastric bypass bariatric procedure, and pyloric exclusion in complex gastro-duodenal perforations. In the past, distal gastrectomy with loop GJ was used for patients with peptic ulcer disease. Due to the advent of acid inhibitory medications, this surgical procedure is rarely done nowadays. However, it is still done at our center for complications like bleeding ulcers. The most common complication following GJ is stenosis, followed by marginal ulcers and perforation [3]. Gastric remnant cancer (GRC) is the gastric carcinoma that develops in the remnant stomach more than five years after distal gastrectomy for benign disease [4-7]. The incidence of GRC ranges from $1 \%$ to $8 \%$ [5]. However, gastric carcinoma after GJ without gastric resection for benign disease is rarely reported in the literature $[2,8]$. 
metaplasia, a precancerous condition. This alkaline medium with intestinal metaplasia allows bacterial colonization, which reduces nitrates in the food into nitrosamines which are carcinogenic and proven to cause gastric carcinoma [2]. Given the longer life span of patients with benign disease, such patients with loop GJ are exposed to reflux for a longer time than patients with malignant etiology. With or without gastric resection, the gastrointestinal continuity can be restored by Billroth-II type loop GJ \pm Braun's enterostomy or Roux-en-Y GJ. In addition to the reduced incidence of other complications, Roux-en-Y GJ has been proved to be associated with decreased incidence of reflux and intestinal metaplasia [5-7]. However, the appropriate length of the Roux-en-Y limb, which can completely prevent reflux, is still under debate. Though some argue for a 40-cm loop, the literature supports a loop length of $60 \mathrm{~cm}$ to obviate jejunal reflux completely [9].

Patients with loop GJ develop carcinoma commonly at the anastomosis site and rarely in the stomach away from the anastomosis site $[5,10]$. The reported time interval between loop GJ and gastric carcinoma diagnosis ranges from 15 years to 25 years, the maximum being 50 years [4,8]. The shortest time interval reported is 10 years after surgery for a duodenal ulcer [8]. In our case, carcinoma was diagnosed around 35 years after the previous surgery. The presentation of carcinoma stomach post-GJ would be the same as in patients without prior GJ; pain epigastric region, hematemesis, and GOO are common symptoms. UGIE would be the first investigation for diagnosis. The stomach should be thoroughly examined along with the anastomotic site and GJ's afferent and efferent loops. Adenocarcinoma is the most common histological type of gastric carcinoma arising post-GJ. Intestinal type is more common than diffuse variant in such patients [5]. However, signet-ring cell carcinoma arising from the previous GJ anastomotic site has been reported [4]. High incidence of jejunal mesenteric lymph nodal metastasis and direct invasion into the colon or mesocolon has been noted due to previous surgery and reformation of new lymphatic routes alongside the jejunal stoma [5-8]. A CECT would further help identify the extent of the tumor and its relation with surrounding organs and distant metastatic disease.

In the case of resectable tumors, subtotal or total gastrectomy with gastro-jejunal anastomotic site resection with a wide margin must be performed $[5,6,8]$. Given the high incidence of metastasis in the jejunal mesentery, en-bloc wide excision of jejunum with mesentery is mandatory $[5,7,8]$. Despite the extensive local disease, curative or en-bloc resection is possible in close to $90 \%$ of cases $[5,6,8]$. Moreover, many authors have endoscopically treated early GRC successfully with minimum complications $[5,6]$. None of the cases reported so far received neoadjuvant chemotherapy as the tumors were diagnosed before metastasis, and surgery was the initial treatment given in the cases with gastric carcinoma post-GJ. Therefore, the role of neoadjuvant therapy is not clear. Given the rarity of GRC or post-GJ carcinoma without gastrectomy, the prognosis of these tumors is still debated. However, numerous studies have reported survival similar to patients with similar stage primary gastric carcinoma [5-7].

Though there are no studies available regarding the screening for gastric carcinoma in patients with loop GJ without gastrectomy, annual screening with biopsy of the suspicious region is recommended in patients at risk for GRC $[5,6,11]$. However, the time period post-GJ for initiation of screening and duration of screening is not clear. Some authors recommend 10 years [11], whereas others recommend that five years interval from surgery is an appropriate time to start endoscopic surveillance [5]. Therefore, a similar recommendation can be made for patients undergoing GJ without gastrectomy for benign pathology, especially given the possibility of endoscopic treatment for early lesions.

\section{Conclusions}

Although rare, gastric malignancy post-GJ for benign etiology can occur. Although these tumors are prone to extensive local disease, curative or en-bloc resection is possible in most cases. Therefore, the survival of these patients is similar to patients with stage-matched primary gastric carcinoma. Routine annual screening with biopsy of the suspicious region is recommended in patients for early diagnosis.

\section{Additional Information \\ Disclosures}

Human subjects: Consent was obtained or waived by all participants in this study. Conflicts of interest: In compliance with the ICMJE uniform disclosure form, all authors declare the following: Payment/services info: All authors have declared that no financial support was received from any organization for the submitted work. Financial relationships: All authors have declared that they have no financial relationships at present or within the previous three years with any organizations that might have an interest in the submitted work. Other relationships: All authors have declared that there are no other relationships or activities that could appear to have influenced the submitted work.

\section{References}

1. Abdi E, Latifi-Navid S, Zahri S, Yazdanbod A, Pourfarzi F: Risk factors predisposing to cardia gastric adenocarcinoma: insights and new perspectives. Cancer Med. 2019, 8:6114-26. 10.1002/cam4.2497

2. Matsushita M, Hajiro K, Okazaki K, Takakuwa H: Gastric cancer occurring at anastomosis after gastrojejunostomy without gastrectomy. Dig Dis Sci. 1998, 43:898-900. 10.1023/a:1018803106386

3. Sampaio-Neto J, Branco-Filho AJ, Nassif LS, Broska AC, Kamei DJ, Nassif AT: Complications related to 


\section{Cureus}

gastric bypass performed with different gastrojejunal diameters. Arq Bras Cir Dig. 2016, 29 Suppl 1:12-4. 10.1590/0102-6720201600S10004

4. Namikawa T, Kawanishi Y, Fujisawa K, et al.: Gastric adenocarcinoma at the anastomotic site 50 years after gastrojejunostomy: a case report. Mol Clin Oncol. 2017, 7:249-51. 10.3892/mco.2017.1309

5. Mezhir JJ, Gonen M, Ammori JB, Strong VE, Brennan MF, Coit DG: Treatment and outcome of patients with gastric remnant cancer after resection for peptic ulcer disease. Ann Surg Oncol. 2011, 18:670-6. 10.1245/s10434-010-1425-1

6. Takeno S, Hashimoto T, Maki K, et al.: Gastric cancer arising from the remnant stomach after distal gastrectomy: a review. World J Gastroenterol. 2014, 20:13734-40. 10.3748/wjg.v20.i38.13734

7. Chan DC, Fan YM, Lin CK, Chen CJ, Chen CY, Chao YC: Roux-en-Y reconstruction after distal gastrectomy to reduce enterogastric reflux and Helicobacter pylori infection. J Gastrointest Surg. 2007, 11:1732-40. 10.1007/s11605-007-0302-0

8. Umezu T, Takeda J, Tanaka T, Koufuji K, Hashimoto K, Kakegawa T: Early gastric cancer following gastrojejunostomy without gastrectomy. Kurume Med J. 1991, 38:187-90. 10.2739/kurumemedj.38.187

9. Collard JM, Romagnoli R: Roux-en-y jejunal loop and bile reflux . Am J Surg. 2000, 179:298-303. 10.1016/s0002-9610(00)00326-3

10. Thorban S, Böttcher K, Etter M, Roder JD, Busch R, Siewert JR: Prognostic factors in gastric stump carcinoma. Ann Surg. 2000, 231:188-94. 10.1097/00000658-200002000-00006

11. Greene FL: Early detection of gastric remnant carcinoma. The role of gastroscopic screening . Arch Surg. 1987, 122:300-3. 10.1001/archsurg.1987.01400150054010 